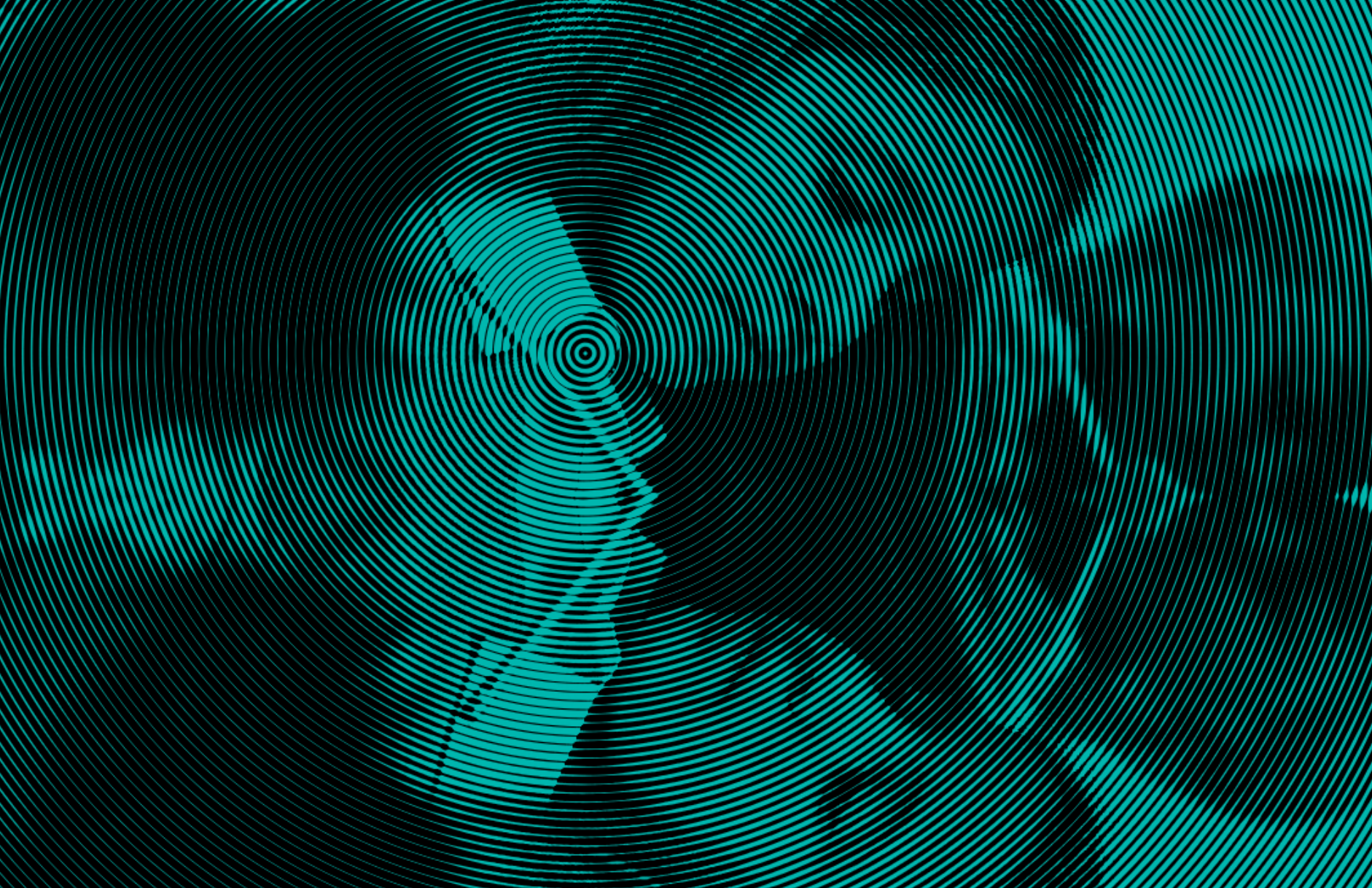

\title{
Los aprendizajes no intencionados en la complejidad del ambiente escolar: asunto emergente y posibilitador de acciones para la paz
}

Not-intentional learning in the complexity of school environment: An emerging matter and actions' facilitator towards peacebuilding

As aprendizagens não intencionadas na complexidade do ambiente escolar: Assunto emergente e possibilidade nas ações para a paz

James Frank Becerra Martínez Oscar Leonardo Cárdenas Forero María Anais Moncada Rodríguez Edith Constanza Negrete Soler Ángela Prieto Acuña Sonia Milena Uribe Garzón 
James Frank Becerra Martínez 1

Oscar Leonardo Cárdenas Forero 2

María Anais Moncada Rodríguez 3

Edith Constanza Negrete Soler 4 Ángela Prieto Acuña 5

Sonia Milena Uribe Garzón 6
1. Colegio Simón Rodríguez IED; Especialista en Pedagogía, Universidad Pedagógica Nacional; Correo electrónico: jafra8@hotmail.com

2. Colegio Entre Nubes S. O.; Magister en Desarrollo Educativo y Social, Universidad Pedagógica Nacional; Correo electrónico: osle1972@gmail.com

3. Colegio Gustavo Restrepo IED; Especialista en Multimedia para la Docencia, Universidad Cooperativa de Colombia; correo electrónico: anais.moncada@gmail.com

4. Colegio Inem Francisco de Paula Santander; Magíster en Enseñanza de las Ciencias Exactas y Naturales, Universidad Nacional de Colombia; correo electrónico: negretesoler@gmail.com

5. Colegio Antonio Baraya; Magister en Educación con Énfasis en Valores y Desarrollo Humano, Universidad Externado De Colombia; correo electrónico: angeis72@yahoo.es

6. Colegio Entre Nubes Sur Oriental; Especialista en Psicología Educativa, Universidad de la Sabana; correo electrónico:sonia.smug80@gmail.com

Maestros en Colectivo

Fecha de recepción: 18 de abril de 2016 / Fecha de aceptación: 16 de septiembre de 2016

\section{Resumen}

En las interacciones del ambiente escolar existen ciertos aprendizajes que no surgen necesariamente de los contenidos curriculares, actividades académicas o intenciones pedagógicas del maestro, sino que involucran formas de lenguaje, comportamientos, actitudes y expresiones no explícitas en esas gestiones, pero visibles en las dinámicas de convivencia de los estudiantes. Estas particulares formas de relacionarse suceden sin la mediación directa de la institución escolar, convirtiéndose en aprendizajes que revisten de gran importancia porque son marcadores de las relaciones de convivencia, potenciadores o inhibidores de los aprendizajes escolares y de los actos de paz en la escuela. Son ellos el punto de partida para el presente artículo.

Palabras clave: Aprendizajes no intencionados, ambiente escolar, interacciones, educación para la convivencia y la paz.

\section{Summary}

There are some interactions, some learning in the school environment that do not appear in relation with curriculum content, academic activities or teacher's pedagogical intentions, but also involve types of languages, behaviors, attitudes and expressions that are not explicit in these activities, but they become visible in the convivial dynamics of the students. These particular ways of relating happen without the direct mediation of the school, becoming learnings that line of great importance in extent that it seems that they are the market of convivial relations, enhancers or inhibitors of the school learning's and the actions to build peace at school.

Keywords: Not-intentional learning, school environment, interactions, peace and coexistence education.

\section{Resumo}

Nas interações que acontecem no ambient escolar, existem certos aprendizados que não necessariamente surgem em relação com os conteúdo currículares, atividades acadêmicas ou as intenções pedagógicas do professor; mas envolvem formas de linguagem, comportamentos, atitudes e expressões que não se encontram explícites nessas atividades, porém se tornam visíveis nas dinâmica de convívencia dos estudantes. Estes modos particulares de relacionarse acontecem sem a mediação directa da instituiçãs, de grande importância na medida em que aparentemente são eles marcadores das relações de convívio, potencializadores ou inibidores da aprendizagem escolar e do ação para a construção da paz na escola.

Palavras chave: Aprendizagens não intencionadas, ambiente escolar, interações, educação para a convivência e paz. 


\section{Presentación}

Una de las intenciones es exponer algunas reflexiones adelantadas por el grupo Maestros en colectivo ${ }^{7}$ alrededor de cómo, en el estudio de las dinámicas, interacciones y tendencias que caracterizan al ambiente escolar, entendido como ese entramado sistémico de actores, actividades y contextos que continuamente se enreda y deshila en el vaivén del tiempo y el espacio de los escenarios escolares, aparecen una serie de aprendizajes no intencionados por los maestros que, en gran medida, determinan, contribuyen y condicionan las maneras de ser sujeto, de relacionarse con los otros y de asumir los conocimientos escolares, y que en ocasiones permanecen dispersos e imperceptibles en el acontecer escolar y en el aula misma, pero que, contrario a ello, se deben considerar para adelantar procesos de formación subjetiva, acciones de paz y relaciones de convivencia distintas, acordes con los requerimientos contemporáneos.

Algunos aprendizajes suelen escapar no solo a las prácticas de enseñanza, sino a la rigurosidad del sistema, y afloran en cualquier momento por efecto de las interacciones que se suscitan entre los agentes escolares, manifestándose como simples preguntas o comportamientos individuales o colectivos que develan que a veces lo enseñado por el maestro trasciende a otros aprendizajes, no intencionales, pero que de igual manera influyen en la formación y en los modos de relación subjetiva.

Estos aprendizajes "no intencionados" (al escaparse la mayoría de veces del protocolo institucional y de la planeación de aula) revisten gran importancia, pues al parecer trascienden hasta la formación convivencial de los estudiantes y ponen en evidencia aquello que con más facilidad se aprende y, ante todo, lo que cautiva los intereses, la construcción de conocimientos y el desarrollo de habilidades y comportamientos en ellos. Entre esos aprendizajes no intencionados aparecen, por ejemplo, situaciones en las que los maestros buscan enseñar a resolver pacíficamente conflictos $y$, sin darse cuenta, lo hacen con acciones inconsistentes en las que se percibe agresión y empleo de la violencia, manifestándoles a los niños que los conflictos son un asunto relacionado con la agresión.

Es importante destacar que el quehacer en la escuela se basa principalmente en prácticas, acciones e intenciones de corte didáctico,

7 Colectivo de maestros de diferentes áreas e instituciones públicas que adelanta estudios e investigaciones alrededor de los Ambientes de Aprendizaje en el Aula, constituido por: María Gilma Acosta Rodríguez, Cristina Behrentz Pfalz, Juan José Garzón. pedagógico y metodológico, pero, sin embargo, en algunas situaciones los objetivos formulados de modo oficial y visible son trascendidos por un entramado de relaciones, saberes, costumbres, conocimientos, imaginarios, sentimientos, emociones, vivencias y conductas que permean el proceso educativo, tanto en su ambiente de mediación cultural como en las otras formas de construir ciudadanía, por la complejidad de las interacciones entre los actores escolares, viéndose afectados y condicionados hacia la incursión de ciertos aprendizajes no intencionados.

Lo anterior implica que, simultáneo a la apropiación y producción de saberes académicos, y en la formación para la convivencia, se desarrollan procesos conjuntos y articulados de socialización con los estudiantes que van más allá de lo que los maestros estructuran en las actividades de enseñanza en el aula, y les dinamizan desde actitudes que fortalecen las dinámicas de convivencia (los conflictos, las tensiones de carácter tribal, religioso, de género, racial, entre otras) desconocidas o no percibidas por el profesorado, olvidando aquello que acontece en relación con la experiencia de principios éticos y estéticos manifiestos en formas de ser, de actuar y de comprender el mundo por parte de los estudiantes, $\mathrm{y}$ que generan experiencias convivenciales colectivas que traspasan lo académico y trascienden en su formación personal.

Esta situación permite ver que al interior de la escuela se posibilitan una serie de aprendizajes vividos como comportamientos, actitudes, acciones, lenguajes y gestos que, a pesar de no ser intencionales para el docente en sus prácticas de enseñanza, intervienen en el proceso de formación académica y ciudadana de los estudiantes. Antes que ocultos, ese conjunto de aprendizajes e influencias hace parte de la cultura escolar, de la convivencia y de las interacciones de los actores escolares que, por alguna razón, pasan desapercibidos y no son reconocidos por no considerarles equivalentes a las actuaciones netamente académicas.

Sin embargo, ese conjunto de aprendizajes circula en las relaciones entre los sujetos y hacen parte de sus códigos, modos de comunicación, de producción de saberes, de interrelación y de construcción de identidad; asuntos fundamentales en la formación política, democrática, ciudadana y para la paz, que de no hacerse evidentes pueden formar subjetividades ajenas a las intenciones de la escuela y, por supuesto, a las apuestas contemporáneas de una sociedad pacífica, respetuosa, democrática y que reconoce la diversidad y la diferencia.

Reconocer estos otros aprendizajes no es sencillo, pero es necesario para dar cuenta de la complejidad de las relaciones subjetivas y, en especial, para aportar elementos de reflexión a la construcción 
de la paz desde la escuela, pues a pesar de estar latentes en su cotidianeidad, en las mentalidades e imaginarios de maestros y estudiantes, y de asumir la forma de ciertas rutinas, paradigmas y prácticas dogmáticas en el aula, condicionando la formación subjetiva y la manera de convivir y relacionarse con los otros, suelen no considerarse al momento de formular alternativas para estudiar las dinámicas convivenciales en la escuela. Si no se cuenta con estrategias para rastrear estos aprendizajes no intencionados, se considerarán solo un asunto de las dinámicas escolares, desconociendo que su reconocimiento es un acto de paz.

En este sentido, una de las estrategias para aproximarse al reconocimiento de los aprendizajes no intencionados, involucra considerar una serie de preguntas: ¿Qué es lo que realmente aprenden los estudiantes?; ¿por qué se aprende?; ¿para qué aprenden eso que aprenden?; ¿cómo es que la estructura biológica y emocional condiciona en determinado momento el aprender?; ¿qué impulsa o motiva a aprender?; ¿el aprendizaje existe en relación directa con el objeto de estudio, o está afectado por el contexto del educando?; ¿cómo aporta la escuela a los procesos de ciudadanía, en el reconocimiento y el respeto por el otro?

Cada una de estas preguntas permitirá a los maestros acercarse a la identificación de estos aprendizajes. Es más, ya en la escena escolar, por ejemplo las formas de organización de los estudiantes para abordar un objeto de estudio, los signos de regulación, actitudes, intereses y disposiciones frente al otro, al igual que las formas de lenguaje pluri-significativo, valores y patrones construidos individual y colectivamente, permiten percibir algunas respuestas a lo planteado que requieren de atención para ver este reconocimiento como un acto que aporta a la paz en el marco de la escuela.

\section{Los aprendizajes, un asunto también emocional}

A menudo, cuando se plantea la necesidad de reflexionar sobre el aprendizaje de los educandos, los maestros suelen hacer afirmaciones a partir de propuestas surgidas de algunas corrientes psicológicas que lo consideran como la captación de algo que es independiente a los sujetos. Sin embargo, pocos se han detenido a examinar que el aprendizaje es un proceso condicionado a la naturaleza y constitución biológica y emocional que poseen como seres vivos.

En este sentido, los sujetos son sistemas determinados con una estructura, y lo que pasa en determinado instante depende de dicha estructura en ese instante (Maturana, 1997), así, el aprendizaje no solo está sujeto al deseo y a la voluntad por saber, sino a aquello que como sistemas biológicos y emocionales nos afecta en determinado momento de la vida, por lo tanto, las personas son sistemas con una estructura de cambio continuo que se afecta y se transforma cotidianamente desde sus miradas y vivencias.

Como se observa, la emoción se convierte en un determinante del aprendizaje, el cual está condicionado por los estados anímicos de los niños y jóvenes, su interés y motivaciones internas y externas o su voluntad y deseo por saber y aprender. Son los estudiantes quienes, dependiendo de esa estructura en constante cambio, determinan qué contenidos culturales han de aprender, desde los puramente académicos, hasta los que son determinantes para desenvolverse en situaciones convivenciales, retadoras, conflictivas y de incertidumbre.

Lo que el estudiante aprende depende de él, de su emoción, de la relación que establezca con los otros y de su deseo, entonces, esta acción es mediada por su estructura interna y no solamente por aquello que procura el maestro para estimularla. Por lo tanto, el aprendizaje es condicionado por la actitud que se asume frente al estudio de algún fenómeno, por esa relación entre el objeto de estudio y la disposición interior.

Curiosamente en ciertas ocasiones los estudiantes no aprenden lo que determinan los propósitos e intenciones pedagógicas, sino aquello que impulsa la emoción o la relación que establecen con el otro, o particularmente lo que desean aprender, quizás "ese asunto" de alguna asignatura escolar que deben memorizar por un momento para luego reproducirlo como para "pasar". Aprenderán aquello que consideren relevante y necesario para sobrevivir con éxito en la escuela, no lo que venga exigido por las peculiaridades de la cultura escolar (Pérez, 1996).

En definitiva, el aprendizaje es totalmente opuesto a captar algo externo, depende de los estudiantes, de si estudian o abordan y posibilitan el conocimiento para solucionar situaciones problema y tomar decisiones. ¿Cómo determinar entonces, si un estudiante sabe o no sabe acerca de algo, si justo en el momento de la actividad escolar su estructura biológica y emocional no está dispuesta y en actitud de aprendizaje?; ¿realmente los estudiantes "no saben"?, ¿’ es que lo que el docente desea enseñar no es de su interés y por eso es innecesario?

Cada uno de estos interrogantes se convierte en punto de partida durante la discusión sobre qué es lo que verdaderamente se quiere que aprendan los educandos, en especial, cuando se enfrentan a una prueba de evaluación de su proceso de aprendizaje o de aquello que 
se cree que aprendieron. $\mathrm{Al}$ respecto, consideremos que lo se hace es escuchar al otro y decidir si éste sabe o no, en tanto satisfaga ciertas nociones respecto de lo que se escucha (Maturana, 1997).

En este sentido, conocer es una adscripción que uno le hace al otro cuando su conducta satisface el criterio de validez que se le da a lo que escucha. Lo cual implica que el aprendizaje es un fenómeno de transformación estructural en la convivencia, no solo de manera congruente se transforma al otro en su estructura, sino en relación con el medio, su historia y su postura, en cuanto a lo que le es benéfico de ello y lo que posiblemente esté en capacidad de obtener. Es por esto que los organismos no se adaptan al medio, sino que en interacción constante se transforman recíprocamente (Maturana, 1997).

Si el aprendizaje depende de cada sujeto, de sus necesidades, intereses, emociones y de aquello que en un momento determinado afecta su estructura biológica y emocional: ¿Es importante que el docente procure que aquello que desea enseñar llame la atención del sujeto, para lograr lo que Maturana denomina el cambio estructural de las personas en la convivencia?; ¿qué es aquello que aprenden los estudiantes y por qué aprenden?; ¿lo qué aprenden apunta a la dialéctica de construcción de conocimiento, o a la repetición de contenidos curriculares? Cuando dentro de sus criterios de evaluación un docente afirma que el estudiante no sabe: ¿A qué hace referencia?; ‘a que no memorizó y divulgó un concepto?; ¿a que no quiso hacer nada en su clase?; ¿a que a pesar de que lo intentó, no logró los niveles esperados? o ¿a que su interés y actitud no estuvieron centrados en la temática abordada por el docente?

Cuando se interroga a los estudiantes acerca de lo que aprenden en la escuela, extrañamente hacen referencia a conceptos de las áreas de conocimiento, pero: ¿es eso lo que realmente aprendieron?; ¿sirve acaso para sus vidas?, o tal vez no mencionan -o no logran divulgar porque no son conscientes de ello- lo que realmente aprenden de la escuela para su desempeño personal y social, y para la construcción de una sociedad democrática, plural y para la paz.

Cuando un niño o un joven ingresa a la escuela no es el mismo sujeto que termina el año escolar, no es el mismo después del descanso, de una clase o de una salida pedagógica, luego de ello es eso que aprende y que logra en la interacción, es el cambio en la estructura de su organismo como ser vivo lo que le invita a seguir transformándose y creando nuevos mundos y formas de relación para emprender acciones de paz. El aprendizaje es un fenómeno de transformación en la convivencia; el tipo de convivencia que se dé en los ambientes de aprendizaje, en el aula y en la escuela misma, favorece ciertas tendencias, comportamientos, procesos de ciudadanía y, por lo tanto, las actitudes y formación para la paz.

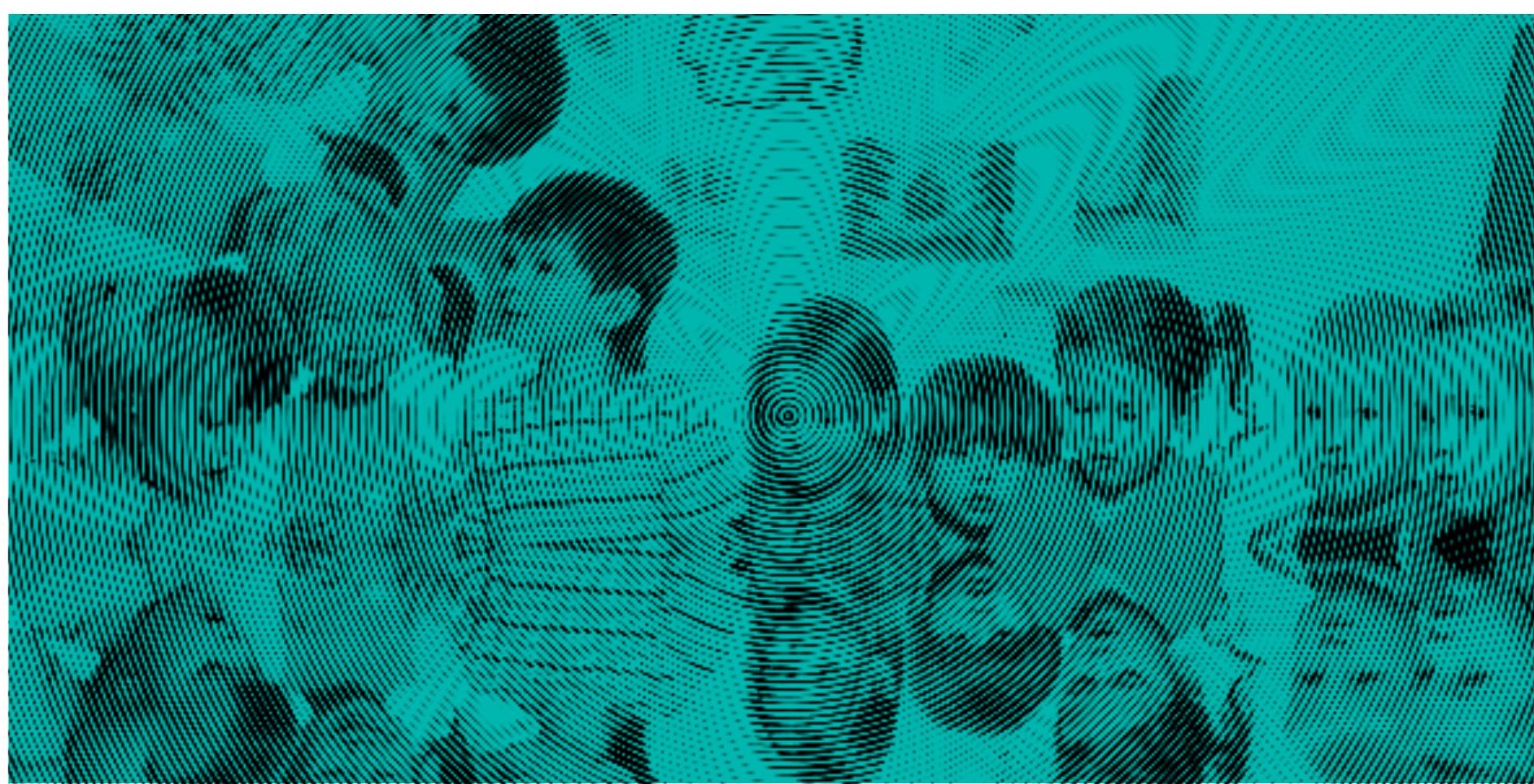




\section{Reconociendo los otros aprendizajes}

Volviendo a los planteamientos iniciales, mediante los discursos y prácticas explícitas e implícitas de los profesores, y de los demás miembros que conviven en la escuela, se logran ciertos aprendizajes, comportamientos, significados e imaginarios en los estudiantes, de los cuales no son plenamente conscientes pero que, sin embargo, hacen parte de la cultura escolar, de la convivencia y de la formación de los sujetos que constituirán una sociedad más equitativa, democrática, plural y pacífica.

En cada actividad escolar, izadas de bandera, festivales, foros, ferias, salidas pedagógicas, juegos, en los descansos, etc., se viven reglas y patrones comunicados explícita o implícitamente que, al no ser entendidos, llevan a los alumnos al riesgo de enfrentar sanciones, afectando no solo la forma de vincularse con el otro, sino las relaciones de convivencia que se establecen al interior de la institución y que transcienden a las esferas privadas y públicas.

De este modo, aquellos estudiantes que ingresan por primera vez a una institución escolar, por lo general se ven comprometidos a leer, validar, confrontar y asumir ciertos roles, comportamientos, lenguajes, pautas y aprendizajes para lograr su permanencia, reconocimiento y, ante todo, su validación como personas que pertenecen a un grupo particular; deben entender los discursos de sus profesores, escoger las respuestas y preguntas pertinentes, apropiadas, aceptables, participar en clase cuando el momento lo exija; de esta forma pueden acomodarse en el contexto del aula de clase, encajando, no corriendo el riesgo del rechazo, de la exclusión o la estigmatización

Mediante las interacciones, en especial las no verbales, con los adultos y, por tanto, con el profesorado, cualquier niño o niña comprende que si presta atención, y se preocupa por atender a lo que los demás realizan, puede ser el centro de interés de un adulto (Torres, 1996). Esto significa que si la escuela en su conjunto educa, los maestros lo hacen con sus actos, con ellos se convierten en modelos para seguir o para rechazar, pues algunos solo despiertan apatía y desinterés, y es en esta dinámica relacional que el aprendizaje se determina, en virtud de la emoción y del agrado por el docente o los mismos compañeros de curso; pensemos en ese curso al cual nos desagrada ir por los comportamientos de los estudiantes, eso es lo que invita a aprender.

No solo se educa con el discurso, también con la expresión, la vitalidad o desgano que se imprime a lo que se hace, con los gestos y actitudes. Inconscientemente el docente establece normas

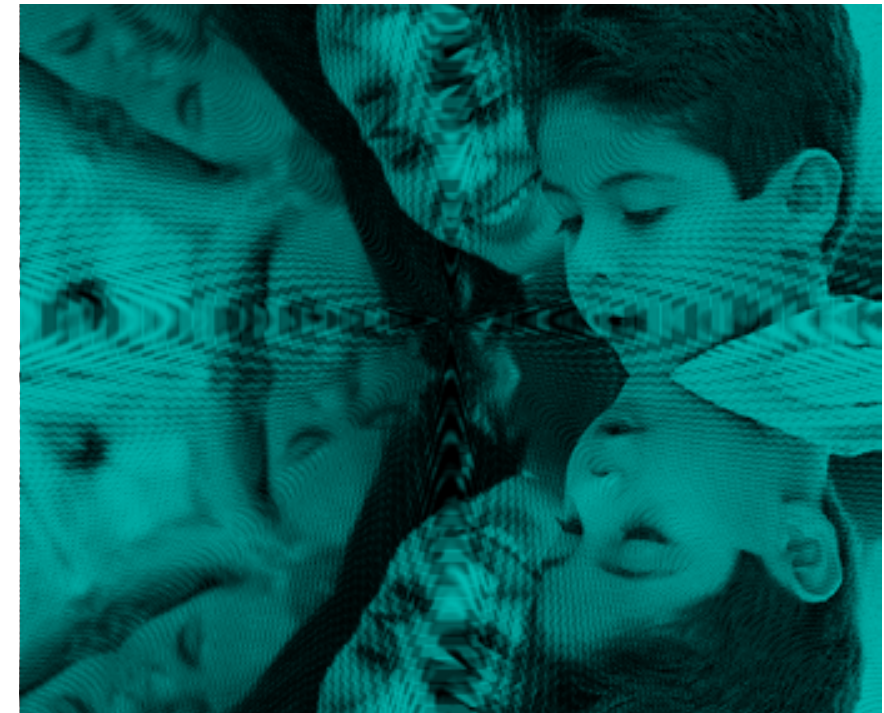

y patrones de comportamiento y son los estudiantes los primeros en evidenciar esa coherencia entre su discurso (teoría) y su actuación cotidiana (práctica). Es mediante la interacción social que se produce a diario la forma como se van construyendo los significados de los objetos y de las situaciones en las aulas, las subjetividades y las habilidades, conocimientos, valores, comportamientos, actitudes de convivencia y destrezas privilegiadas por la sociedad (Torres, 1996).

Sin embargo, aún persisten prácticas escolares que confirman formas particulares de relación con los niños, niñas y jóvenes, maneras de interacción que están condicionadas por la idea de un docente poseedor del conocimiento y unos estudiantes que necesitan aprender mediante la enseñanza de una serie de contenidos propios de las áreas. Ante esta situación queda la inquietud sobre los aprendizajes no intencionales del docente que fueron adquiridos por los alumnos.

Además de esos aprendizajes intencionales, relacionados con las asignaturas escolares, en los que los estudiantes construyen sus formas particulares de representar el conocimiento, de comprender y relacionarse con el mundo, hay otros que son inherentes a la convivencia y a la socialización humana, en los que se aprenden actitudes, normas, comportamientos, lenguajes y gestos que condicionan su interacción con nosotros (maestros y/o directivos) y con el entorno, escolar y extra-escolar.

Estos otros aprendizajes, pedagógicamente hacen parte de la cultura escolar y, en ocasiones, generan en los estudiantes actitudes que los definen como sujetos con capacidades excesivamente 


\section{Referencias}

Apple, M. (1995). Educación y poder. Barcelona: Paidós.

Cazden, C. (1991). La interacción entre iguales: procesos cognoscitivos. El discurso en el aula. El lenguaje de la enseñanza y el aprendizaje. Barcelona: Paidós.

Maestros en Colectivo. (2006). Ambientes de aprendizaje en el aula. Bogotá: IDEP.

Maturana, H., y Varela, F. (1997). El sentido de lo humano. Chile: Dolmen.

Ministerio de Educación Nacional (MEN). (1998). Lineamientos curriculares en Educación Ética y Valores Humanos. Bogotá: MEN.
Pérez, Á. (1996). La cultura escolar en la sociedad neoliberal. Málaga: Morata.

Segura, D. (2002). La enseñanza de las ciencias en Colombia. Revista Innovación y Ciencia, Vol. X. No. 3-4. Bogotá.

Suárez, R. (1994). Educación y sociedad. La educación. Bogotá: Trillas.

Torres, J. (1996). El curriculo oculto. Madrid: Morata.

Trilla, J. (1993). La escuela y el medio. Una reconstrucción sobre el contorno de la institución escolar. Barcelona: Ariel. 